\title{
Oximetry Alone Versus Portable Polygraphy for Sleep Apnea Screening Before Bariatric Surgery
}

\author{
Maude Malbois • Vittorio Giusti • Michel Suter • \\ Cyril Pellaton • Jean-Frédéric Vodoz $\cdot$ Raphael Heinzer
}

Received: 26 July 2009 / Accepted: 1 December 2009/Published online: 6 January 2010

(C) Springer Science+Business Media, LLC 2009

\begin{abstract}
Background Screening for obstructive sleep apnea (OSA) is recommended as part of the preoperative assessment of obese patients scheduled for bariatric surgery. The objective of this study was to compare the sensitivity of oximetry alone versus portable polygraphy in the preoperative screening for OSA.

Methods Polygraphy (type III portable monitor) and oximetry data recorded as part of the preoperative assessment before bariatric surgery from 68 consecutive patients were reviewed. We compared the sensitivity of $3 \%$ or $4 \%$ desaturation index (oximetry alone) with the apnea-hypopnea index (AHI; polygraphy) to diagnose OSA and classify the patients as normal ( $<10$ events per hour), mild to moderate ( $10-30$ events per hour), or severe ( $>30$ events per hour).

Results Using AHI, the prevalence of OSA (AHI $>10$ per hour) was $57.4 \%: 16.2 \%$ of the patients were classified as severe, $41.2 \%$ as mild to moderate, and $42.6 \%$ as normal.
\end{abstract}

\footnotetext{
M. Malbois

University of Lausanne,

Lausanne, Switzerland

V. Giusti

Division of Endocrinology, Diabetology and Metabolism, Department of Internal Medicine, CHUV,

Lausanne, Switzerland

M. Suter

Department of Surgery, Hôpital du Chablais,

Aigle-Monthey, Switzerland

C. Pellaton $\cdot$ J.-F. Vodoz $\cdot$ R. Heinzer $(\triangle)$

Center for Investigation and Research in Sleep (CIRS)

and Pulmonary Department,

Lausanne University Hospital (CHUV),

BH06 204, 1011 Lausanne, Switzerland

e-mail: rheinzer@post.harvard.edu
}

Using 3\% desaturation index, $22.1 \%$ were classified as severe, $47.1 \%$ as mild to moderate, and $30.9 \%$ as normal. With $4 \%$ desaturation index, $17.6 \%$ were classified as severe, $32.4 \%$ as mild, and $50 \%$ as normal. Overall, $3 \%$ desaturation index compared to AHI yielded a $95 \%$ negative predictive value to rule out OSA (AHI $>10$ per hour) and a $100 \%$ sensitivity $(0.73$ positive predictive value) to detect severe OSA (AHI $>30$ per hour).

Conclusions Using oximetry with 3\% desaturation index as a screening tool for OSA could allow us to rule out significant OSA in almost a third of the patients and to detect patients with severe OSA. This cheap and widely available technique could accelerate preoperative work-up of these patients.

Keywords Bariatric surgery · Sleep apnea - Type III portable monitor · Oximetry Lung volume · Preoperative work-up . Oxygen desaturation

\section{Introduction}

Obstructive sleep apnea (OSA) is a common disease with a prevalence of $4 \%$ in middle-aged men and $2 \%$ in middle-aged women [1]; however, only a limited portion of these patients are diagnosed and treated. OSA is commonly encountered in morbidly obese patients undergoing bariatric surgery with a prevalence of $76 \%$ to $96 \%$, but only $15 \%$ to $19 \%$ of these patients have an established diagnosis of OSA [1-3]. There is an increasing amount of evidence suggesting that sleep apnea has a significant negative impact on both the perioperative and postoperative complications in these patients [2, 4-7]. Sedatives, analgesics, and anesthetics alter upper airway tone, and airway obstruction and death have been reported in patients with OSA. Obese patients with 
OSA usually have a narrow upper airway due to fat deposition inside the pharyngeal walls (so-called fat pads), which result in a natural tendency for the upper airway to collapse [8]. During wakefulness, these obese patients need to actively keep their upper airway open by contracting their upper airway dilator muscles, which allows them to breathe normally. When they fall asleep, these muscles relax and the upper airway tends to collapse, yielding an apnea or a hypopnea. When carbon dioxide and oxygen levels reach a certain threshold, the patients arouse, which allows them to reactivate their upper airway dilator muscles. If patients receive benzodiazepine premedication or opiates after surgery, the main risk they are exposed to is prolonged oxygen desaturation due to a delayed arousal at the end of the apnea or to a more severe collapse of the upper airway due to the myorelaxing effects of these drugs. It is, therefore, important to diagnose and treat OSA preoperatively.

Screening for OSA is recommended as part of the preoperative assessment of the patients by the Bariatric Scientific Collaborative Group and the International Federation for the Surgery of Obesity Guidelines for Safety, Quality, and Excellence in Bariatric Surgery. A multidisciplinary team that includes pulmonologists or sleep specialists is, therefore, recommended to evaluate every patient scheduled for bariatric surgery. In addition to the routine preoperative assessment for major abdominal surgery, the patient scheduled for bariatric surgery should undergo further assessment that includes sleep apnea syndrome screening [9, 10].

Limited channel home monitoring (type III portable monitors measuring nasal airflow, chest and abdominal movements, and pulse oximetry) or pulse oximetry alone are used in Europe to screen patients for OSA before surgery. Oximetry alone is an easier and less costly way to perform this screening compared to using portable monitors, but its sensitivity and specificity in morbidly obese patients is yet unknown. The objective of this study was thus to compare the sensitivity of oximetry alone versus portable polygraphy in the preoperative screening for OSA in this population.

\section{Materials and Methods}

Screening of Medical Records and Patient Selection

Medical records of 68 obese patients (23 males and 45 females) who underwent bariatric surgery by Roux-en-Y gastric bypass between November 2006 and June 2008 at the University Hospital of Lausanne (CHUV) were retrospectively reviewed. All patients were evaluated by a multidisciplinary team, including an endocrinologist, a psychologist, a dietician, an anesthesiologist, a pulmo- nologist, and a bariatric surgeon. The indication for bariatric surgery was considered if their body mass index (BMI) exceeded $40 \mathrm{~kg} / \mathrm{m}^{2}$, or $35 \mathrm{~kg} / \mathrm{m}^{2}$ with at least one comorbidity related to their weight, and after failure of conservative treatment for at least 2 years.

\section{Anthropometric Measures}

Body weight and height were measured with a Detecto scale and stadiometer, and the BMI was calculated as weight (in kilograms) divided by the square of the height (in square meters).

\section{Respiratory Assessment}

Sleep apnea screening was performed with an Embletta system (Embla, Broomfiels, CO, USA). This system incorporates nasal pressure measurements to estimate airflow, thoracic and abdominal bands for measuring respiratory effort, and finger pulse oximeter recordings. The recorder is tied to the chest with a Velcro band and also detects body position. The pulse oximeter (Nonin, Plymouth, MN, USA) has a sampling rate of $10 \mathrm{~Hz}$. All data were analyzed by the same investigator using the "Chicago criteria" for the definition of apnea and hypopnea [11]. Desaturation was defined as a $3 \%$ or $4 \%$ drop in oxygen saturation from the pre-event baseline. Oxygen drop had to occur within a 1-min time frame. The apnea-hypopnea index (AHI) was defined as the total number of apnea + hypopnea occurring per hour of sleep. The oxygen desaturation index (ODI) represents the number of oxygen desaturation occurring per hour of sleep.

Data collected from these recordings include AHI, 3\% oxygen desaturation events (OD3\%), 4\% oxygen desaturation events (OD4\%), average desaturation amplitude (AD\%), lowest oxygen saturation (LOD), average oxygen saturation (AOS\%), supine AHI, nonsupine AHI, supine time (in percent), nonsupine time (in percent), obstructive, central, and mixed apnea frequency, and proportion of time spent with an oxygen saturation $<90 \%$ and $<80 \%$.

Patients were connected to the monitoring equipment at the hospital sleep center in the late afternoon, slept at home, and brought the recorder back the following day. At least $5 \mathrm{~h}$ of good quality data including at minimum oxygen saturation and flow was required to validate the recording.

Table 1 Demographic data

\begin{tabular}{lrrc}
\hline & Mean & SD & Range \\
\hline Age (year) & 39.4 & 10.3 & $18-61$ \\
BMI $\left(\mathrm{kg} / \mathrm{m}^{2}\right)$ & 44.3 & 5.1 & $37.3-61.9$ \\
Weight $(\mathrm{kg})$ & 123.5 & 19.7 & $81.3-192.2$ \\
\hline
\end{tabular}


Table 2 Summary of statistical data for polygraphy and oximetry measurements in our sample population

\begin{tabular}{lccc}
\hline & Mean & SD & Range \\
\hline Apnea hypopnea index (AHI) & 15.3 & 13.8 & $0.6-53.9$ \\
Supine AHI & 21.5 & 25.0 & $0-142.5$ \\
Nonsupine AHI & 11.8 & 12.47 & $0-59.8$ \\
Supine time (\%) & 37.0 & 25.9 & $0-99.8$ \\
Obstructive apnea (\%) & 6.3 & 10.7 & $0-62.5$ \\
Central apnea (\%) & 1.9 & 5.8 & $0-38.1$ \\
Mixed apnea (\%) & 0.5 & 1.7 & $0-11.8$ \\
Hypopnea index (\%) & 13.5 & 11.5 & $0.4-41.1$ \\
OD per hour $\geq 3 \%$ & 21.7 & 17.9 & $1.3-69.1$ \\
OD per hour $\geq 4 \%$ & 15.6 & 14.9 & $0.6-55.1$ \\
Lowest saturation (mean \%) & 82.0 & 8.4 & $51-94$ \\
Saturation $<90 \%$ (\% time) & 10.3 & 21.1 & $0-99.4$ \\
Supine OD per hour 3\% & 27.2 & 24.7 & $0-127$ \\
Nonsupine OD per hour 3\% & 18.2 & 17.3 & $0-69.2$ \\
Supine OD per hour 4\% & 19.1 & 23.11 & $0-149.5$ \\
Nonsupine OD per hour 4\% & 11.6 & 13.17 & $0-56.5$ \\
\hline
\end{tabular}

Otherwise, the recording was repeated on a subsequent night. The investigator analyzed the data using all the recorded channels and reanalyzed the data for all patients using only the oximetry recording.

For each patient, we compared the sensitivity of OD3\% or OD4\% (oximetry alone) with AHI (polygraphy) to diagnose OSA and classify the patients as normal $(<10$ events per hour), moderate (10-30 events per hour), or severe ( $>30$ events per hour). We also compared time spent in supine and nonsupine posture with oxygen saturation parameters (LOD, saturation $<90 \%$, saturation $<80 \%$, $\mathrm{OD} 3 \%$, and $\mathrm{OD} 4 \%$ ).

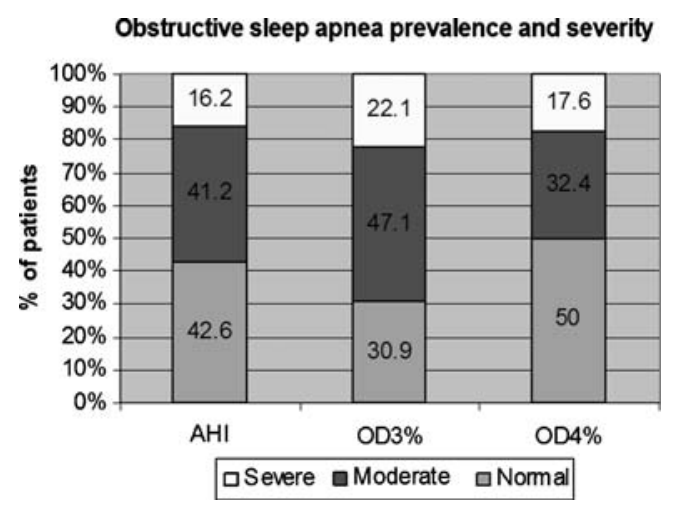

Fig. 1 OSA prevalence in our population as determined by ambulatory polygraphy (apnea + hypopnea per hour $=A H I$ ) or oximetry alone $(3 \%$ or $4 \%$ desaturation per hour $=O D 3 \%$ and OD4\%). Normal ( $<10$ per hour), mild to moderate (10-30 per hour), severe $(>30$ per hour $)$
Table 3 Oximetry sensitivity, specificity, PPV, and NPV for an AHI $>10$ per hour

\begin{tabular}{lcc}
\hline & OD3\% & OD4\% \\
\hline Sensitivity (\%) & 97 & 83 \\
Specificity (\%) & 69 & 93 \\
PPV (\%) & 81 & 94 \\
NPV (\%) & 95 & 79 \\
\hline
\end{tabular}

Statistical Analysis

All analyses were performed using the Jump 7 statistical package (The SAS Institute, Carry, NC, USA) running on a PC. Means and standard deviations (SD) were calculated for age, anthropometric measurements, and all respiratory variables. Comparison of the sensitivity of ODI versus AHI was assessed by analysis of variance and paired Student's $t$ test. Spearman's correlation was used to assess relationships between polygraphy and oximetry results and different anthropometric parameters. Differences were interpreted as statistically significant when $p<0.05$.

\section{Results}

Sixty-eight obese patients (23 males and 45 females) scheduled to undergo bariatric surgery were included in this study (anthropometric and demographic data are displayed in Table 1).

Using AHI (portable monitor), $16.2 \%$ of the patients were classified as severe ( $>30$ events per hour), $41.2 \%$ as mild to moderate (10-30 events per hour), and $42.6 \%$ as normal $(<10$ events per hour; Table 2). Using OD3\%, $22.1 \%$ of the patients were classified as severe, $47.1 \%$ as mild to moderate, and $30.9 \%$ as normal. With OD4\%, $17.6 \%$ were classified as severe, $32.4 \%$ as mild to moderate, and $50 \%$ as normal (Fig. 1).

Overall, OD3\% compared to AHI yielded a 95\% negative predictive value (NPV) to rule out OSA (AHI> 10 per hour) and a $100 \%$ sensitivity ( 0.73 positive predictive value $[\mathrm{PPV}]$ ) to detect severe OSA (AHI>30 per hour; Tables 3 and 4). The concordance coefficient was

Table 4 Oximetry sensitivity, specificity, PPV, and NPV for an AHI $>30$ per hour

\begin{tabular}{lrc}
\hline & OD3\% & OD4\% \\
\hline Sensitivity (\%) & 100 & 91 \\
Specificity (\%) & 93 & 96 \\
PPV (\%) & 73 & 83 \\
NPV (\%) & 100 & 98 \\
\hline
\end{tabular}




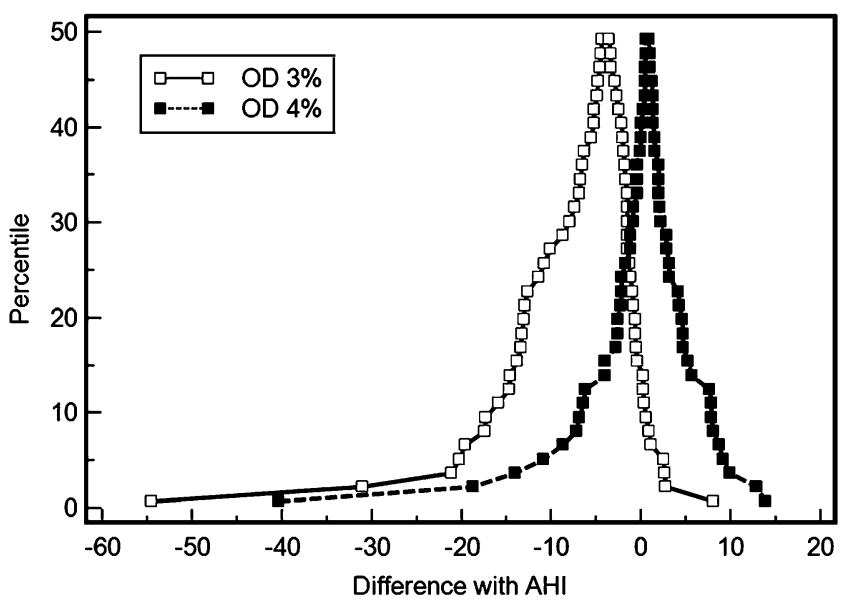

Fig. 2 Mountain plot showing the difference between AHI and 3\% or $4 \%$ ODIs $(O D 3 \%, O D 4 \%)$

0.759 (CI, 0.661-0.832) between AHI and OD3\% and was 0.856 (CI, 0778-0.908) between AHI and OD4\%. As shown on the mountain plot in Fig. 2, OD4\% and OD3\% distributions are both very close to AHI and to one another.

Among the correlations (Table 5), BMI was not correlated with AHI or with ODI.

\section{Discussion}

The main finding of this study is that oximetry alone with a $3 \%$ desaturation threshold was able to rule out significant OSA (defined as an AHI $>10$ per hour) in $30 \%$ of morbidly obese patients scheduled for bariatric surgery, with a NPV of $95 \%$. Overall, the prevalence of OSA (defined as AHI $>10$ per hour) in our population was $57.4 \%$, which is lower than the proportion reported in previous studies on patients undergoing bariatric surgery where a prevalence of $70 \%$ to $95 \%$ of OSA was reported $[3,5]$. However, when we used a broader definition of OSA (AHI $>5$ per hour), $72.7 \%$ of our patients would be positive for OSA, which corroborates previous findings.

Various strategies have been proposed for screening sleep apnea before surgery, ranging from polysomnography (complete sleep study in a sleep laboratory), the most expensive and time-consuming test, to a simple costless questionnaire [12]. In Europe, portable polygraphy (type III portable monitors) is the most common test used to screen OSA before surgery, but recommendations may vary from one country to another. Compared to oximetry, portable polygraphy is able to detect more subtle breathing disturbances such as a hypopneas or short apnea (not associated with oxygen desaturation) since it provides direct measurements of respiratory efforts and flow [13]. It is important to detect these types of subtle respiratory events when patients are investigated for daytime sleepiness or fatigue since even mild respiratory events may cause repetitive arousals, which can alter sleep quality and induce daytime sleepiness. However, when a patient is screened for OSA before surgery, it is not clear which parameter (AHI or ODI) is the most accurate predictor of perioperative complications. Anesthetics, sedatives, and opiates, which are often used in the postoperative period, tend to alter upper airway muscles tone, decrease the "respiratory drive," and increase the arousal threshold, thereby allowing longer sleep apnea and more severe oxygen desaturation to occur. We can thus speculate that patients who experience the most severe oxygen desaturation during the screening night will be more susceptible to oxygen desaturation during and after surgery.

Considering that oximetry alone has a $95 \%$ NPV to rule out OSA among the morbidly obese patients included in our study, this technique may provide enough information to classify patients as low risk if the ODI is below 10 per hour $(30.9 \%$ of our patients) or as high risk if their ODI is
Table 5 Summary of correlations between the different variables

\begin{tabular}{llcc}
\hline Variable & By variable & Spearman $\rho$ & $p$ value \\
\hline Apnea hypopnea per hour (AHI) & Age & 0.342 & 0.004 \\
Apnea hypopnea per hour (AHI) & Weight & 0.183 & 0.125 \\
Supine time (\%) & Weight & -0.345 & 0.004 \\
OD per hour $>3 \%$ & Age & 0.429 & $<0.001$ \\
OD per hour $>3 \%$ & Weight & 0.264 & 0.03 \\
OD per hour $>4 \%$ & Age & 0.460 & $<0.001$ \\
OD per hour $>4 \%$ & Weight & 0.299 & 0.01 \\
Lowest OD $(\%)$ & Age & -0.466 & $<0.001$ \\
Lowest OD $(\%)$ & Weight & -0.179 & 0.144 \\
Saturation $<90 \%(\%)$ & Age & 0.484 & $<0.001$ \\
Time with saturation $<90 \%$ & Weight & 0.229 & 0.061 \\
Average desaturation $(\%)$ & Weight & 0.384 & 0.001 \\
\hline
\end{tabular}


$>30$ per hour (severe OSA in $22.1 \%$ of our patients). According to our results, $53 \%$ of the patients $(30.9 \%$ without OSA and $22.1 \%$ with severe OSA) could thus be screened by oximetry alone, which would save time and expense. Therefore, only patients with an intermediate ODI (i.e., between 10 and 30 per hour) should still be investigated with a portable polygraphy, but this category represents less than half $(47 \%)$ of our study population. However, before any type of screening strategy can be recommended, a prospective randomized study will be needed to determine which OSA screening approach, if any, translates into a reduction of adverse events in surgical patients undergoing bariatric surgery.

We were surprised by the high sensitivity of oximetry with an OD3\% threshold to detect OSA in the population we studied. In the general population, pulse oximetry alone lacks sensitivity [14] because hypopnea and short apnea may not induce a sufficiently large oxygen desaturation to be detected by this device. However, in the morbidly obese population we studied, oximetry with a OD3\% threshold was as sensitive as AHI to diagnose significant OSA defined as an AHI>10 per hour. Our hypothesis is that, in morbidly obese patients, even mild apnea or hypopnea induces oxygen desaturation because abdominal fat reduces lung volume, thereby decreasing the thoracic oxygen reserve. This reduction in lung volume causes oxygen saturation levels to drop faster during respiratory events compared to lean individuals with a normal lung volume. Moreover, this lung volume reduction is likely exacerbated in supine posture due to a rostral shift of the diaphragm, which is pushed upwards by abdominal pressure, causing a further reduction in lung volume [4]. In addition, it has been demonstrated in animals and humans that a reduction in lung volume increases upper airway collapsibility due to a decrease in mediastinal traction on pharyngeal walls [15]. This hypothesis is supported by a significant correlation between weight and AHI as well as OD3\% and OD4\% rates (Table 5), which suggests that, the more obese the patients were, the more oxygen desaturation they experienced. Weight was negatively correlated with the percentage of time spent supine. This may be due to a greater respiratory discomfort for the most obese patients because they have more respiratory events in a supine position. BMI was not correlated with AHI or ODI. This surprising finding was previously reported by O'Keefe et al. who screened 170 morbidly obese patients with polysomnography [5]. Age was also positively correlated with the respiratory disturbance indexes. This is easily explained by the increase in OSA severity with age which has been reported in many epidemiological studies [16, 17$]$.

There are a few limitations to this study. First, full polysomnography, which is recognized as the gold standard for the diagnosis of OSA, was not performed on these patients; oximetry results were thus compared with a limited channel portable monitor. However, several studies have shown that portable monitors yield results close to polysomnography [18-20] and we thus believe that our results would still be valid if oximetry was compared to full polysomnography. Second, this study does not address the outcome of these patients according to the screening method because the design of the present study did not allow us to do so. This important question should be addressed in a future prospective randomized study. Finally, these results only apply to morbidly obese patients and cannot be generalized to other populations undergoing surgery.

In conclusion, using oximetry with $\mathrm{OD} 3 \%$ as a screening tool for OSA could allow us to rule out significant OSA in almost a third of morbidly obese patients during preoperative work-up for bariatric surgery. This cheap and widely available technique could accelerate preoperative work-up of these patients by avoiding long waiting lists for full-night (ambulatory or in-lab) studies. Patients with an intermediate oximetry result (OD 10-30 per hour) should, however, undergo further sleep investigations to confirm the diagnosis. Patients complaining of severe daytime sleepiness should be referred to a sleep specialist even when oximetry is negative.

Conflict of Interest The authors declare that they have no conflict of interest.

\section{References}

1. Young T, Plata M, Dempsey J, et al. The occurrence of sleepdisordered breathing among middle-aged adults. N Engl J Med. 1993;328:1230-5.

2. Hallowell PT, Stellato TA, Schuster M, et al. Potentially lifethreatening sleep apnea is unrecognized without aggressive evaluation. Am J Surg. 2007;193:364-7.

3. Lopez PP, Stefan B, Schulman CI, et al. Prevalence of sleep apnea in morbidly obese patients who presented for weight loss surgery evaluation: more evidence for routine screening for obstructive sleep apnea before weight loss surgery. Am Surg. 2008;74:834-8.

4. Benumof JL. Obstructive sleep apnea in the adult obese patient implications for airway management. J Clin Anesth. 2001;13:14456.

5. O'Keefe T, Patterson EJ. Evidence supporting polysomnography before bariatric surgery. Obes Surg. 2004;14:23-6.

6. Ballantyne G, Svahn J, Capella R, et al. Predictors of prolongated hospital stay following open and laparoscopic gastric bypass for morbid obesity: body mass index, length of surgery, sleep apnea, asthma and the metabolic syndrome. Obes Surg. 2004;14:104250.

7. Kaw R, Aboussouan L, Auckley D, et al. Challenges in pulmonary risk assessment and perioperative management in bariatric surgery patients. Obes Surg. 2008;18(1):134-8. 
8. Schwab RJ, Pack AI, Gupta KB, et al. Upper airway and soft tissue structural changes induced by CPAP in normal subjects. Am J Respir Crit Care Med. 1996;151:1106-16.

9. Melissas J. IFSO guidelines for safety, quality and excellence in bariatric surgery. Obes Surg. 2008;18:497-500.

10. Fried M, Hainer V, Basdevant A, et al. Interdisciplinary European guidelines for surgery for severe (morbid) obesity. Obes Surg. 2007;17:260-70.

11. American Academy of Sleep Medicine Task Force. Sleep-related breathing disorders in adults: recommendations for syndrome definition and measurement technique in adults. Sleep. 1999;22:667-89.

12. Chung F, Yegneswaran B, Liao P, et al. Validation of the Berlin questionnaire and American Society of Anesthesiologists checklist as screening tools for obstructive sleep apnea in surgical patients. Anesthesiology. 2009;110(1):194.

13. Hussain SF, Fleetham JA. Overnight home oximetry: can it identify patients with obstructive sleep apnea-hypopnea who have minimal daytime sleepiness? Respir Med. 2003;97(5):5537-40.
14. Jobin V, Mayer P, Bellemare F. Predictive value of automated oxygen saturation analysis for the diagnosis and treatment of obstructive sleep apnoea in a home-based setting. Thorax. 2007;62(5):379-80.

15. Heinzer RC, Stanchina ML, Malhotra A. Lung volume and continuous positive airway pressure requirements in obstructive sleep apnea. Am J Respir Crit Care Med. 2005;172(1):114-7.

16. Lévy P, Pépin JL, Malauzat D, et al. Is sleep apnea syndrome in the elderly a specific entity? Sleep. 1996;19(3Suppl):S29-38.

17. Palla A, Digiorgio M, Carpenè N, et al. Sleep apnea in morbidly obese patients: prevalence and clinical predictivity. Respiration. 2008;78:134-40.

18. Thurnheer R, Bloch KE, et al. Respiratory polygraphy in sleep apnoea diagnosis. Swiss Med Wkly. 2007;137:97-102.

19. Douglas NJ. Home diagnosis of the sleep apnoea/hypopnoea syndrome. Sleep Med Rev. 2003;7(1):53-9.

20. Kingshott RN, Vennelle M, et al. Predictors of improvements in daytime function outcomes with CPAP therapy. Am J Respir Crit Care Med. 2000;161(3 Pt 1):866-71. 